\title{
Geometric Landmark-based Pose Tracking for Holonomic Mobile Robots
}

\author{
Hamid Taheri \\ Nanjing University of Science and Technology \\ NJUST
}

\author{
Zhao Chun Xia \\ Nanjing University of Science and Technology \\ NJUST
}

\begin{abstract}
Wheeled Mobile Robot (WMR) navigation is subjected to noise that brings several factors of uncertainties in the robot's motion positioning as well as pose tracking. The uncertainty in motion makes the robot's pose-tracking to be a difficult task. Motion models have been designed to track the robot poses within trajectories based on the robot's inertial velocitybased information or the odometry readings obtained from its wheel encoders. This paper introduces a holonomic motion model based on geometric relationships between a robot's laser-scanner and the landmarks in the environment. The laser-scanner is attached to an Omnidirectional Mobile Robot (OMR) capable of holonomic drive and thus the observationbased motion-tracking approach can capture the trajectory obtained of holonomic motions as well. In this method, despite the pioneering methods, the pose information in the laser-base-frame is used instead of the robot's inertial information. In this way, a different perspective of sensing is provided that can enhance the robot's pose tracking task. The method can be used along with a common robot's motion model to further reduce the uncertainties in motion. The experiment is conducted based on a KUKA Youbot equipped with a Hokuyo URG-04lx laser range scanner in its front that suggests that the method can successfully track the robot's trajectory with a good level of consistency in restricted areas where the landmark distribution has covered the whole trajectory.
\end{abstract}

\section{Keywords}

Motion Model; Laser scanner; Localization; Landmark tracking; Geometric pose tracking

\section{INTRODUCTION}

Mobile robots in distinction with robots with fixed-base platforms operate in more unrestricted workspaces. Mobile robots are composed based on wheels, trains, legs, and etcetera or based on the combination of several locomotion devices [1]. Wheeled Mobile Robot (WMR) is a common mechanism used in a broad range of applications. A variety of different wheel mechanisms have been utilized in mobile robot designs which among them omnidirectional wheels also known as multidirectional wheels have attracted a wide range of studies. Utilizing an omnidirectional wheel can provide a mobile robot with a holonomic drive able to drive in any direction without requiring a change in the robot's heading direction. A well-detailed study on different types of Omnidirectional WMR (OMR) is available in our previous work [2]. Utilizing four Mecanum wheels [3] in a car-like configuration results in achieving a stable holonomic drive. A study on a four Mecanum wheel-based car-like configuration is available in our previous work [4]. OMR pose tracking is challenging even though the motion without changing the bearing enhances the pose tracking, but the fact is that the robot in the real world would have a change in direction as well as changes in its rotation anyway. The robot's pose tracking usually is done within motion models which the majority of them are designed based on non-holonomic WMRs. The existing approaches use either robot's inertial velocity-based information or the odometry readings obtained from the wheel encoders and the uncertainty in motion is usually dealt with in noise processing in sampling stages using methods such as probabilistic motion models in SLAM and localization frameworks based on different methods such as: particle filters [5], Kalman filters [6], Extended Kalman filters (EKF), unscented KF (UKF) [7], adaptive UKF (AUKF) [8], Markov chain Monto Carlo [9], etc. Different methods involving different areas of studies have been conducted to deal with uncertainty in mobile robot navigation uncertainty, for instance, heuristic [10], non-heuristic methods [11], nonlinear approximating methods [12] and Neural Network (NN) [13] Fuzzy logic approaches [14], [15], etc.

Localization [16] and pose-tracking, in general, the relative navigation is a challenging task especially because of the existence of noise in robot's motion coming from systematic and non-systematic errors existing in robot's mechanism and the environment's dynamic nature. The majority of researches has been focused on the effects of the dynamic factors, noise, and conditions on the system where they try to improve the accuracy of robots observation under uncertainties the assumption of most methods is that the motion model is obtained and the observation is supposed to overcome the errors in motion model. Moreover, most studies have been conducted regardless of the differences in the characteristic nature of different motion in different types of WMR locomotion mechanisms. Furthermore, the Light Detection and Ranging (LIDAR) laser scanners have been used to determine the locations of landmarks and to establish the map of the environment with respect to robot's poses. In this paper, the description of a geomatic motion model based on the relationships between the landmarks distributed in the environment and the robot's laser scanner is presented. The sensory readings are taken to determine the robot's poses rather than determining the locations of the landmarks as it is usually done in observation models. In this way, a measurement on the robot's trajectory obtained through a third source of sensing is available that can be used along with the robot's motion model to enhance the robot's positioning. However, the noise formation and inertial-based motion model design are out of this scope, this study shows that tracking the sensor's information can result in obtaining a robot's trajectory. Figure 1 shows a schematic of such systems that both perspectives of sensing is used. The laser-based motion tracking along with robot's inertial based pose tracking can be both used in a motion model to decrease the uncertainty. Section 2 mathematically describes the geometric relationships among the robot and the landmarks to establish the robot's pose tracking. Section 3 shows a trajectory obtained in experiments using a KUKA Youbot WMR and 
section 4 is the conclusion and future works.

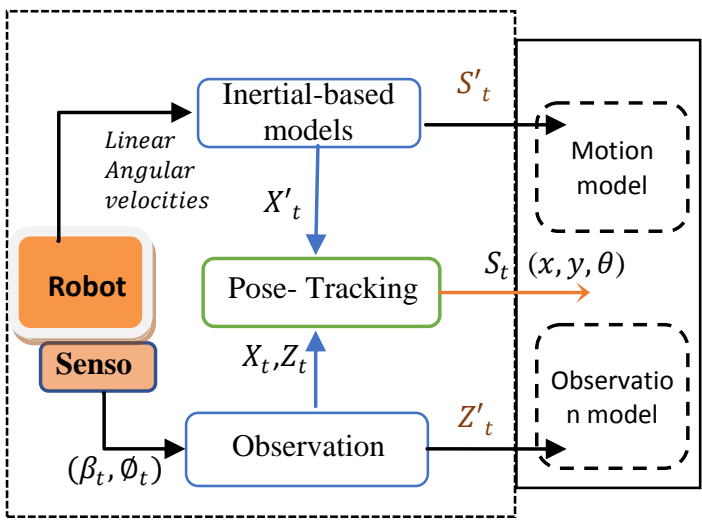

Fig 1: System schematic.

\section{LASER-BASED POSE TRACKING}

\subsection{System description}

In our system, the robot is equipped with four Mecanum wheels (see Figure 2) capable of holonomic omnidirectional motion. A Hokuyo URG-04lx laser range scanner is attached to the front side of the robot's base platform. Hokuyo laser is capable of scanning 240-degrees with a maximum radius of 4 (m) in each beam, however, due to the configuration of the sensor in front of the robot 180-degrees of observations is available on the system.

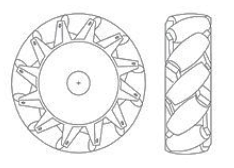

Fig 2: Mecanum wheel schematic.

To track the robot's positions, the method simply takes six angles in the account (see Figure 3) according to the robot's motion direction and the availability of the landmark in that range. And thus, only an available landmark in the related range will be used in the calculations. There are several methods (i.e. triangulation) for landmark detection and tracking in literature, simply a definition of a priority to choose a landmark is used and then by calculation the differences in the angles and ranges, the system obtains the robot's locations and the amount of robot's movement in respect to the landmarks.

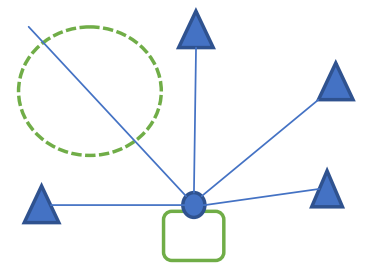

Fig 3: Landmark location possibilities.

The priority is the beam at the range of $\tau / 2$ beam for longitudinal movement, 0 or $\tau$ degree for the transversal drive, and $\tau / 4$ for orthogonal movement obtained from a simultaneous longitudinal and transversal drive.

Moreover, the motion model based on velocity or odometry information is out of the scope of this paper but it's assumed to be partially available.

\subsection{Pose Tracking}

To keep track of the robot's motion the approach tracks the distance between the robot's laser scanner and the landmark's location. The priority of choosing a landmark for tracking the laser-pose is the ones in the direction of the robot's motion direction.

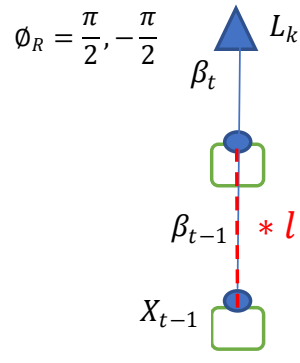

(a)

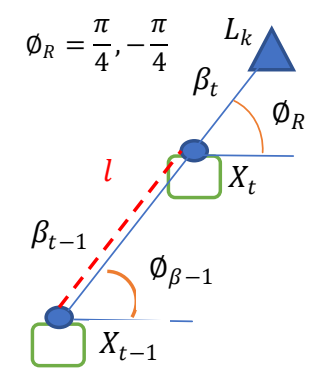

(b)
$\emptyset_{R}=\pi,-\pi$

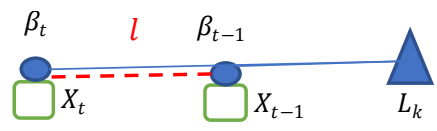

(c)

Fig 4: Landmarks in motion directions

In case of motions towards the landmark, in other words, when $\emptyset_{\beta-1}=\emptyset_{\beta}$ (see Fig. 4), the length of motion $l$ can be measured directly by measuring the length of the beam towards the landmark as follow:

$$
\begin{gathered}
l=\beta_{t-1}-\beta_{t} \\
Z_{t}=Z_{t-1}+\left(\begin{array}{c}
l \cos \emptyset_{R} \\
l \sin \emptyset_{R} \\
0
\end{array}\right)
\end{gathered}
$$

Where $\beta_{t}$ is the laser beam at time t, $\emptyset_{R}$ is the angle of the beam and $\boldsymbol{Z}_{\boldsymbol{t}}<\mathrm{x}_{\mathrm{t}}, \mathrm{y}_{\mathrm{t}}, \theta_{\mathrm{t}}>$ is the robot pose obtained based on the observation in the laser-frame as well. To compute the robot's angle at each measurement the measured $\mathrm{x}$ and $\mathrm{y}$ are used as follow:

$\theta_{\mathrm{t}}=\tan ^{-1}\left(\frac{\mathrm{y}_{\mathrm{t}}}{\mathrm{x}_{\mathrm{t}}}\right)$

When the landmarks are not available at front of the direction (Figures 5 and 6 (a) parametrization to (b)) or if the robot's motion is curve motion rather than a straight forward motion towards the landmark, in other words, if $\emptyset_{\beta-1}<>\emptyset_{\beta}$, the system still can measure the robot's position in coordinate with the landmark. In general, if the location of a landmark is known the one can calculate the robot's pose using the sensory data and the landmark's information by having $X_{R}=\left(x_{r}, y_{r}\right)_{t}, L_{k}=\left(L_{x}, L_{y}\right)_{t}, z_{t}=\left(\beta_{t}, \emptyset_{t}\right)$.

$x_{t}=L_{x}-\beta_{t} \cos \emptyset_{R}$

$y_{R}=L_{y}-\beta_{t} \sin \emptyset_{R}$

$X_{R}=L_{k}-\beta_{t} \cdot\left[\begin{array}{l}\cos \emptyset_{R} \\ \sin \emptyset_{R}\end{array}\right]$

Where $X_{R}$ is the robot's location with respect to a landmark in $X_{R} R Y_{R}$ frame. To transform the pose from the laser-frame coordinate to world coordinate the approach does as follow: 
${ }^{R} T_{W}=\left[\begin{array}{l}\sin \theta_{w} \\ \cos \theta_{w}\end{array}\right]$

$X_{t}=X_{R} \cdot{ }^{R} T_{W}$

Suppose that a detected landmark's location is not known and $\emptyset_{\beta-1}<>\emptyset_{\beta}$, the estimation of the robot's position is obtained by taking the relation between two observations at two states in account.

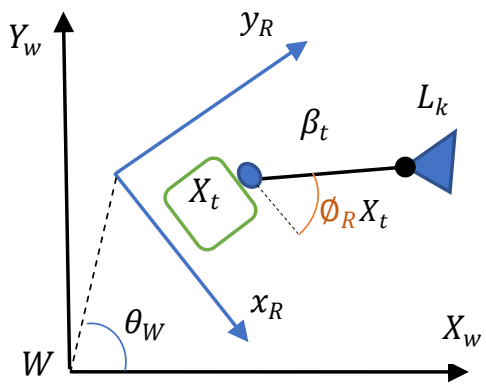

Fig 5: Landmarks location in robot-frame and robot location in world-frame $X_{w} W Y_{w}$.

We define two approaches according to $\emptyset_{R}$ the angle between two observations at two states, first when $\emptyset_{R}>90^{\circ}$ and the second approach for $\emptyset_{R} \leq 90^{\circ}$.

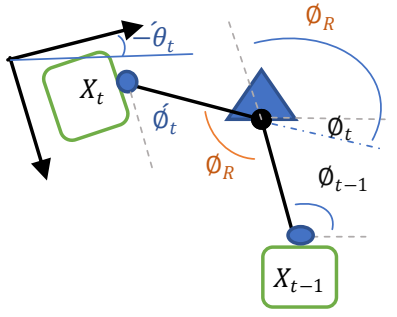

(a)

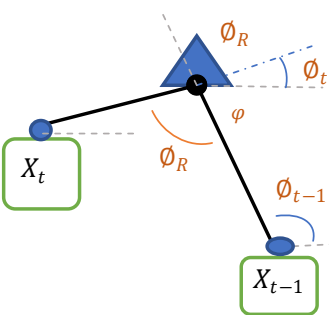

(b)
Fig 6: A location in different robot poses and the gap.

The idea is that when $\emptyset_{R}>90^{\circ}$ it covers a wider area around the landmark, so it is more likely for the robot to meet a known landmark by which can make a more reliable tracking of its pose, and also it consequently does a more reliable detection on the new landmark. And to obtain $\emptyset_{R}$ the following relation is utilized:

$\emptyset_{R}=\emptyset_{t-1}-\emptyset_{t}$

Where the angle of the sensor's signal in world coordinate $\varnothing$ at each time step can be transformed from $\emptyset_{t}$ the robot's coordinate by applying the following equation:

$\emptyset_{t}=\emptyset_{t}+\theta_{t}$

When $\emptyset_{R}>90^{\circ}$ more informative parametrizations can be obtained as shown in figure 7 :

$x^{\prime}=k+o$

$y^{\prime}=v-i$

$k=\beta_{t} \cos \emptyset_{t}$

$o=\beta_{t-1} \sin \varphi$

$v=\beta_{t-1} \cos \varphi$

$i=\beta_{t} \sin \emptyset_{t}$

We also can measure $l$ the traveled distance, by applying the law of cosines as follow:

$$
\begin{aligned}
& l^{2}={\beta_{t}}^{2}+\beta_{t-1}{ }^{2}-2 \beta_{t} \cdot \beta_{t-1} \operatorname{Cos} \emptyset_{R} \\
& l=\left|\sqrt{l^{2}}\right|
\end{aligned}
$$

By having these parameters, the system can make efficient estimations and measurements on the poses.

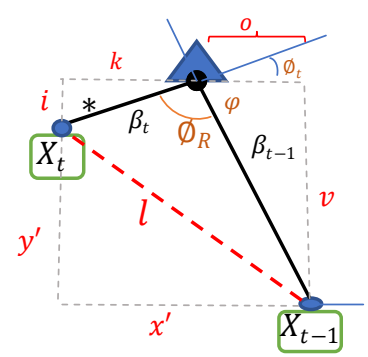

Fig 7: Extend parametrization of location-relations

Firstly, the location of a new landmark $L^{*}{ }_{k}$ can be estimated using the known robot's pose or from a pose obtained through a motion model and a motion correction obtained from the previous states of the robot.

$L_{k \mid t-1}^{*}=X_{t-1}+\left[\begin{array}{l}o \\ v\end{array}\right] \cdot{ }^{\mathrm{R}} T_{W}$

$L_{k \mid t}^{*}=X_{t}+\left[\begin{array}{c}k \\ i\end{array}\right] \cdot{ }^{\mathrm{R}} T_{W}$

$\varepsilon_{L}=L_{k \mid t-1}^{*}-L_{k \mid t}^{*}$

$L_{k \mid t-1}^{*}$ is the new landmark detected at t-1 and $L_{k \mid t}^{*}$ is the landmark detected at $\mathrm{t}, \varepsilon_{L}$ is the error representing the difference between these two observations. $\varepsilon_{L}<>0$ means that the landmark's location is with error and one of the following three conditions may be true: first, one of the observations is correct $L_{k \mid t-1}^{*}$ or $L_{k \mid t}^{*}$. To figure out if this condition is true, the system may compute the robot's poses using the measured landmark's information to find out which one is correct. If a correct measure is found then the system chooses the correct measurements for $L_{k}^{*}$.

$$
\begin{aligned}
& X_{t-1}=L_{k \mid t-1}^{*}-\left[\begin{array}{l}
0 \\
v
\end{array}\right] \\
& X^{\prime \prime}{ }_{t-1}=L_{k_{\mid t}}^{*}-\left[\begin{array}{l}
0 \\
v
\end{array}\right] \\
& \alpha_{t}=X_{t-1}-X_{t-1} \\
& \alpha^{\prime}{ }_{t}=X_{t-1}-X^{\prime \prime}{ }_{t-1}
\end{aligned}
$$

The measurements only take $X_{t-1}$ in account suppose that the system has had the poses correctly estimated so far. By doing so several assumptions also can be made as follow:

1- If $\alpha_{t}=0$ or $\alpha_{t}^{\prime}=0$ then the system knows that either the observation at $t-1$ or $t$ is correct and so it can use it for $L_{k}^{*}$.

2- If the both variable $\alpha_{t}$ and $\alpha_{t}^{\prime}<>0$ then the system knows that both observations are noisy. On this case, the system assumes that however, it knows that the $L_{k}^{*}$ is noisy but still, it doesn't know if the robot's pose at the t state $X_{t}$ is correct or not, so that the approach takes both parameters in the account and estimates the location of the landmark using both $X_{t-1}$ and $X_{t}$. In this way, if $X_{t}$ were not correctly estimated, it will be corrected in the next state and there the location of $L_{k}^{*}$ also can be re-corrected again. Thus, the following equations are driven: 
$L_{k}^{*}=\frac{L_{k \mid t}^{*}+L_{k \mid t-1}^{*}}{2}$

$\dot{X}_{t-1}=L_{k}^{*}-\left[\begin{array}{l}0 \\ v\end{array}\right]$

$X^{\prime}{ }_{t}=L_{k}^{*}-\left[\begin{array}{l}k \\ i\end{array}\right]$

$\alpha_{t}=X_{t-1}-X_{t-1}$

$\alpha^{\prime}{ }_{t}=X_{t}-X^{\prime}{ }_{t}$

$G=-\frac{\alpha_{t}+\alpha^{\prime} t}{2}$

$X^{\prime \prime}{ }_{t}=\left(L_{k}^{*}+G\right)-\left[\begin{array}{l}k \\ i\end{array}\right]$

$X_{t}=\frac{X_{t}+X^{\prime \prime}}{2}$

$L_{k}^{\prime}=X_{t}+\left[\begin{array}{c}k \\ i\end{array}\right]$

$L_{k}^{\prime \prime}=X_{t-1}+\left[\begin{array}{l}O \\ v\end{array}\right]$

$L_{k}^{*}=\frac{L_{k}^{\prime \prime}+L_{k}^{\prime}}{2}+G$

3- If $\alpha_{t}=0$ and $\alpha_{t}^{\prime}=0$ then: both observations are correct and the error has happened due to the wrong estimation of the $X_{t}$. If observations are more likely to be correct $\left(\varepsilon_{L}=0\right)$, to check the uncertainty in robot's poses the one can use the following relation:

$l^{\prime}=\left|\frac{\sqrt{\left(x_{t}-x_{t-1}\right)^{2}+\left(y_{t}-y_{t-1}\right)^{2}}}{2}\right|$

$\varepsilon_{x}=l^{\prime}-l$

If $\varepsilon_{x}=0$, the method defines the poses of the robot and landmarks as reliable estimations. If $\varepsilon_{x} \leq 0.5$ for a new landmark, it flags the $X_{t}$ pose as an uncertain pose which means this pose cannot be used for another new landmark detection. However, the topology of the environment in the system is in such a way that the robot would not meet two new landmarks in a row, hence, the robot will be able to correct its pose in its next state by using known landmark information. If $\varepsilon_{x}>0.5 \mathrm{~m}$ it means the misallocation is over half meters and probably there is a risk of robot's platform collision or large wheel slippage, in other words, practically the system has entered a critical state and out of usage.

When $\emptyset_{R} \leq 90^{\circ}$, suppose that the landmark location is not known, the system can measure the landmark's position through the second approach. Figure 8 shows two examples where $\emptyset_{R}$ is smaller than 90 degrees.

$$
\begin{aligned}
& L_{k \mid t-1}=X_{t-1}+\left[\begin{array}{l}
\beta_{t-1} \cos \emptyset_{t-1} \\
\beta_{t-1} \sin \emptyset_{t-1}
\end{array}\right] \\
& L_{k \mid t}=X_{t}+\left[\begin{array}{l}
\beta_{t} \operatorname{Cos} \emptyset_{t} \\
\beta_{t} \sin \emptyset_{t}
\end{array}\right] \\
& L_{t-1}=L_{k \mid t-1} \cdot{ }^{R} T_{W} \\
& L_{t}=L_{k \mid t-1} \cdot{ }^{R} T_{W}
\end{aligned}
$$

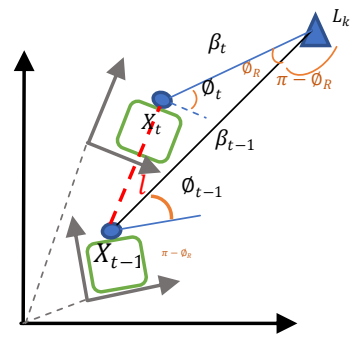

(a)

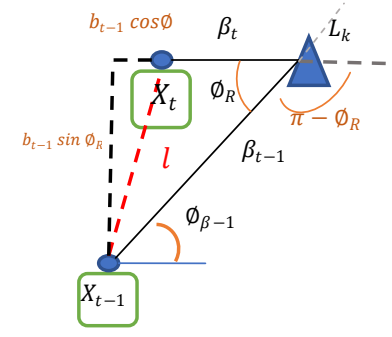

(b)
Fig 8: location-Relation possibilities when $\emptyset_{\mathbf{R}}<90$.

By having landmark $L_{k}$ being observed at two different states a stronger belief can be made. One way is to simply take the average value between these two observations on the same landmarks, in case if one of the observations were not accurate:

$\dot{L}_{k}=\frac{L_{t-1}+L_{t}}{2}$

Then by computing $X_{t}$ robot's location according to $L_{k}$ the new observed landmark's location and $Z_{t}$ the beams from the laser range finder, it can be estimated that which one of the observations had come with a better measurement:

$\dot{X}_{t}=\dot{L}_{k}-\beta_{t-1} \cdot\left[\begin{array}{c}\cos \emptyset_{t} \\ \sin \emptyset_{t}\end{array}\right]$

$\dot{X}_{t-1}=\dot{L}_{k}-\beta_{t-1} \cdot\left[\begin{array}{c}\cos \emptyset_{t-1} \\ \sin \emptyset_{t-1}\end{array}\right]$

With $\alpha_{t}=\dot{X}_{t}-\dot{X}, \alpha_{t-1}=\dot{X}_{t-1}-X_{t-1}$, the system can decide with comparing their differences:

$$
f(x)=\left\{\begin{array}{l}
L_{t-1}, \alpha_{t-1}<\alpha_{t} \\
L_{t}, \alpha_{t}<\alpha_{t-1} \\
K . L_{k}, \alpha_{t}>0.5 \text { and } \alpha_{t-1}>0.5 \text { (Distorted) }
\end{array}\right.
$$

$\mathrm{K}$ is the gain in case if both observations had over $0.5(\mathrm{~m})$ differences which means either the observations are false or the position estimated by the motion model is false, in other words, the system would know that in this case a nonsystematic uncertainty by some other factors, either related to the robot platform such as wheel slippage, drift, etc. has happened or the beams from the landmark are distorted (i.e. glassy landmarks). If the information of such landmarks were important, the system could deal with it by flagging the landmark as a distorted landmark and use some sampling algorithms to identify a better belief which that would be out of the scope of this paper. In this paper, the landmarks are not a priority to this project so that the system can isolate the possible uncertainty in robot's position and define $\mathrm{K}=0$, in other words, it deleted the landmark and let the uncertainty of the robot's location to be dealt with in next states where the robot would meet some known landmarks.

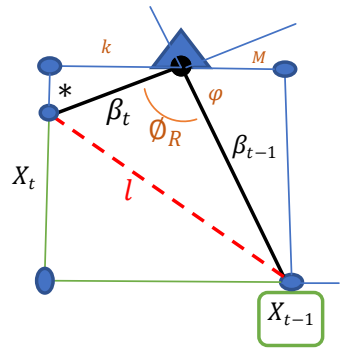

(a)

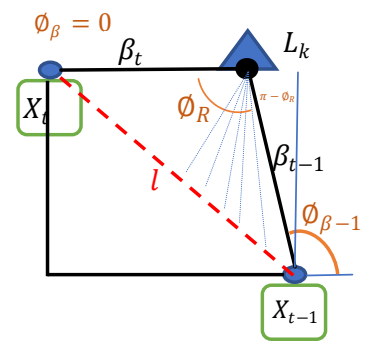

(b)
Fig 9: Observations when $\emptyset_{\mathrm{R}} \leq 90^{\circ}$ and $\emptyset_{\mathrm{b}} \approx 0$ 
Furthermore, in this approach, the landmarks are taken the same as the tables or the spots landmarked with special objects where the robot is supposed to meet at each stop, however in case of larger environments with less number of landmarks where not all motions would have an available landmark to use as its coordinator for position correction using the measurement, the robot may still be able to track its positioning by solely using the motion model until it eventually detects an available landmark, then the system will correct the robot's movements according to the detected landmark under the condition that the motion uncertainty will not be more than a meter, the measurement uncertainty will not be over $0.5(\mathrm{~m})$ and the position of the essential landmarks related to robot's task trajectory are known.

Moreover. places without landmarks can be assumed to be known if there are known landmarks in other observable locations. In other words, not having any available landmarks at a specific space can be considered as an abstract landmark that can still give some information about the robot's approximate location as well. This idea is especially useful to locate the robot where it is confused between several poses.

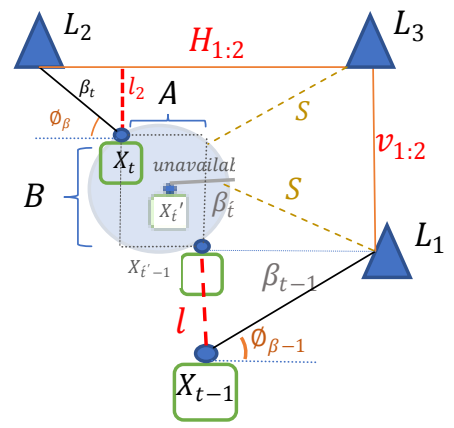

Fig 10: Pose relations in coordination to landmarks.

$S$ represents the ranges that would be available to the robot. It indicates that if the robot were in the $S$ range, it could detect the landmark $L_{3}$. With $S$ an approximation of the place where surely robot is not located can be obtained. Having a close look at figure 10 , one can predict that the robot's more likely position is in the area $(A \times B)$ that can provide the system with the information that determines the robot's location at the time $X_{\hat{t}}^{\prime}$ is surely in $(A \times B)$ area and thus if the robot's pose is estimated out of this area an error in robot's motion has happened that can be dealt with in the same manner as it's described earlier in this paper. A and B can simply be calculated from $X_{t}$ and $X_{t-1}$ considering the robot pose on its path $X_{t-1}^{\prime}$ to $X_{t}^{\prime}$. However, in a more reliable approach, in cases where a prior map exists, it's possible to define this kind of area in the map as abstractive landmarks.

\section{EXPERIMENTS}

To experiment with the described approach, a Hokuyo URG04lx laser scanner is attached to the front fame of a KUKA Youbot OMR base-platform (see Figure 11). The robot is equipped with four Mecanum wheels which makes it to be capable of the holonomic drive towards 8 directions as shown in Figure 12, and the laser is capable of scanning 240-degrees with $4(\mathrm{~m})$ radius range of beams.

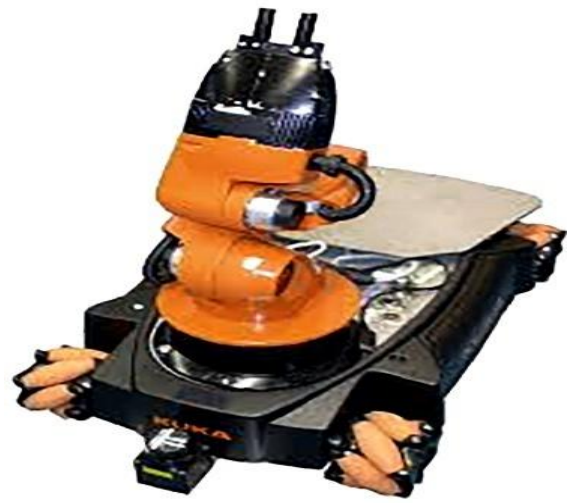

Fig 11: OMR platform.

The workspace is a static $6 \times 6$ meters indoor lab environment with a flat even surface. There are several cylinder-shaped landmarks manually distributed in known locations as well as unknown landmarks. The robot is driven to 14 stops with 16 motion commands. A pre-movement to the navigation is executed to calibrate the robot and to tune the motion gains. Note that the calibration is not in the scope of this paper and the experiments cover the navigation after the robot had set on its 0,0 origin. The starting point of the navigation is taken as the center of the robot's world; however, the mapping is not covered in this experiment.

The approximate motion and directions of the robot is graphically indicated in Figure 12.

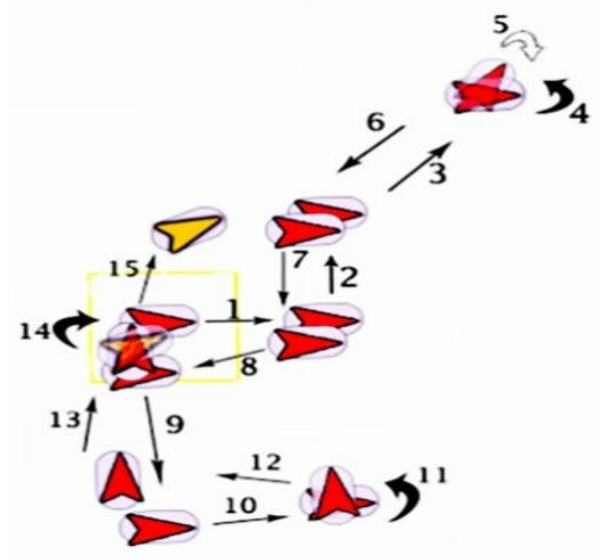

Fig 12: OMR approximate trajectory.

Figure 13 shows the robot's actual trajectory along with approximate locations of priorly known landmarks.

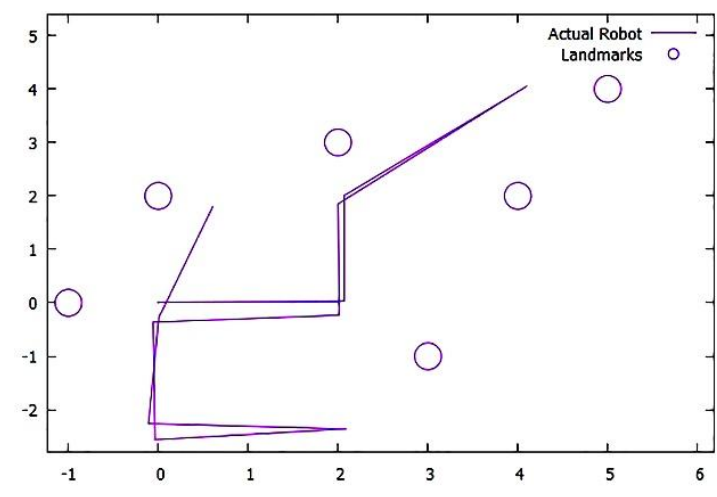

Fig 13: Robot's Actual trajectory. 
Figure 14 shows the poses obtained by the pose-tracking method. The circles indicate the center of landmarks, note that the exact diameter of the landmarks may differ with the real objects used in the experiments. In this test, only the robot's pose is in our interest.

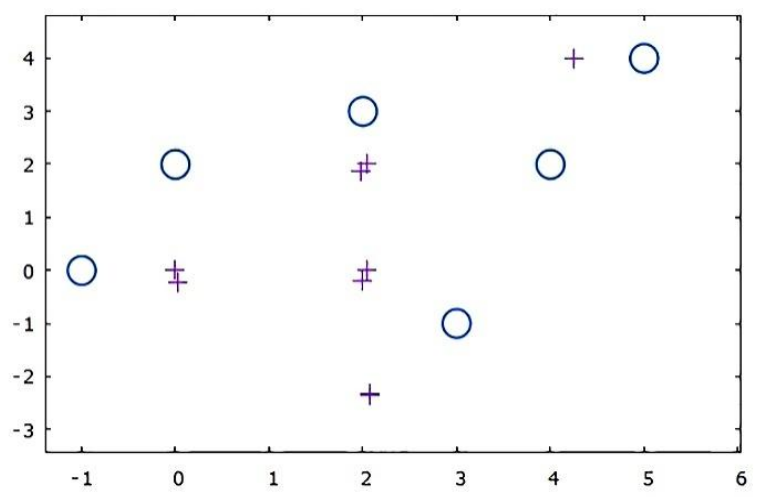

Fig 14: OMR approximate trajectory.

Figure 15 shows the robot's poses along with the actual trajectory that the robot has traveled. Note that the smoothing and trajectory correction due to the overall pass is not carried in the primitive motion model, that may cause differences in pose-to-pose distances that need to be dealt with. Moreover, as it's indicated there may be several poses where the robot would either confuse between similar landmarks facing data association problem or no existed landmarks neither an abstractive landmark is recognized (see table 1). For this reason, once the robot's pose is computed based on its inertial-based velocity, the information can be used for such conditions to obtain a trajectory as in Figure 16.

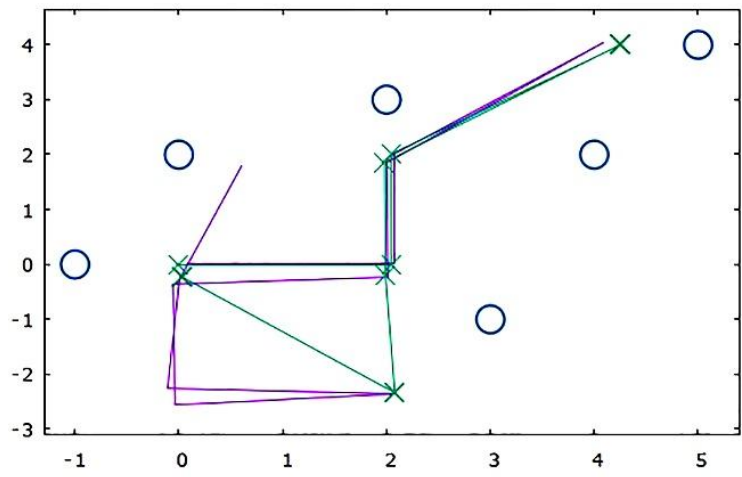

Fig 15: OMR approximate trajectory.

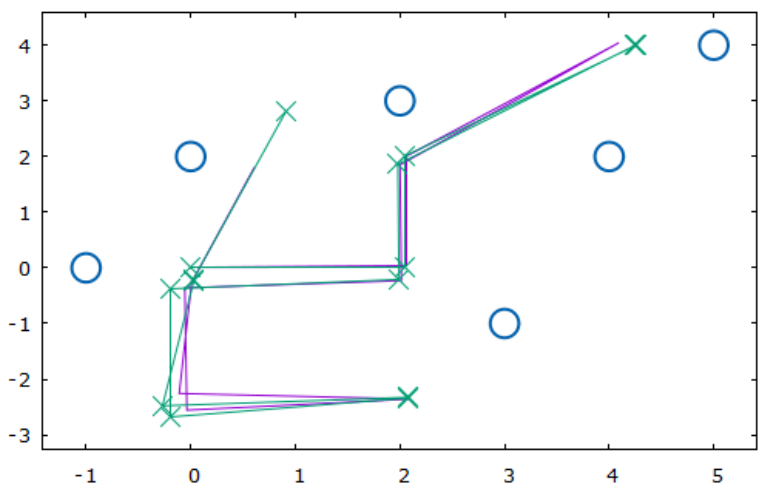

Fig 16: OMR approximate trajectory.

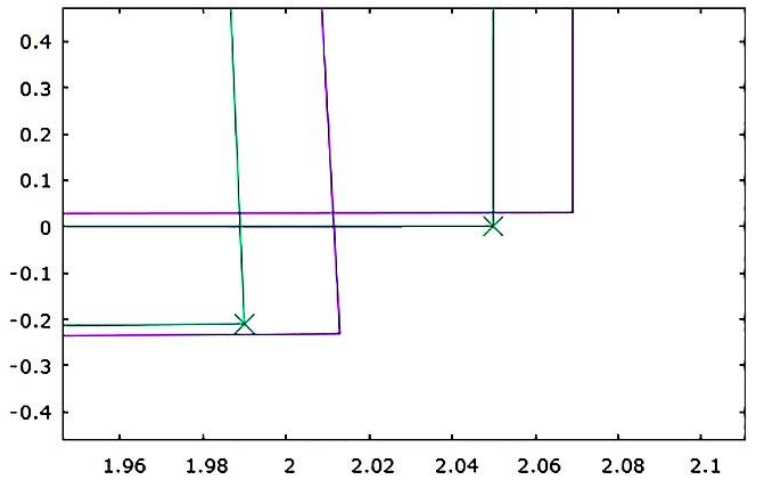

Fig 17: OMR approximate trajectory.

Table 1. Numeric values.

\begin{tabular}{|c|c|}
\hline Actual robot & Pose tracking \\
\hline $2.07,0.03,0$ & $2.05,0,0$ \\
\hline $2.07,2.011,0$ & $2.05,2.02,0$ \\
\hline $4.091,4.043,0$ & $4.26,4,0$ \\
\hline $4.091,4.043,57.2958$ & $4.25,4,01,54.2$ \\
\hline $4.090,4.043,0.0936$ & $4.25,4,01,0.07$ \\
\hline $2.000,1.85,0.0936$ & $1.98,1.86,0.09$ \\
\hline $2.013,-0.23,0.0936$ & $1.99,-0.21,0.09$ \\
\hline$-0.057,-0.36,0.094$ & - \\
\hline$-0.037,-2.56,0.0936$ & \\
\hline $2.096,-2.36,0.0936$ & $2.08,-2.34,0.09$ \\
\hline $2.096,-2.36,-57.3894$ & $2.078,-2.33,-54.5$ \\
\hline $2.096,-2.358,-114.592$ & $2.07,-2.33,-113.6$ \\
\hline$-0.11,-2.258,114.684$ & \\
\hline $0.01,-0.226,-114.52$ & $0.03,-0.23,-114.5$ \\
\hline $0.02,-0.226,-57.2958$ & $0.02,-0.22,-56.96$ \\
\hline $0.605,1.796,-57.296$ & - \\
\hline & \\
\hline & \\
\hline
\end{tabular}

The measurements still include some level of uncertainty due to some amount of distortion in laser beams as well as the differences in detecting the landmark's center-of-gravity measured in different locations. However, as it's shown in Figure 17, the errors are in a reliable range.

\section{CONCLUSION AND FUTURE WORK}

Mobile robot navigation is subjected to noise. Motion models are designed to obtain robot pose and trajectory based on velocity or odometry information obtained from the robot's inertial information. This paper mathematically indicated a detailed geometric pose tracking based on the information obtained from a laser-scanner. The method locates the laser within the environment in contrast to the common observation models that are responsible to locate the landmarks. The laserframe is considered as a part of the robot's base frame that results in obtaining the robot's pose within the presented 
method. However, there are still several aspects that can be further studied. For instance, the landmark detection has a significant role in this approach that can be studied in detail. Moreover, the offered pose-tracking method can be used in a pose-correction method along with another source of sensing such as the robot's traditional motion models. In future work, we tend to design a controller to decide between estimated poses obtained through an odometry-model and the poses obtained from the laser-based pose tracking method to obtain a more accurate robot trajectory. As the experiments are conducted, the method can be successfully utilized in indoor environments, however, by expanding the landmark detection task extending to unknown landmark detection, the method could be utilized in more complex spaces.

\section{REFERENCES}

[1] L. Bruzzone and G. Quaglia, "locomotion systems for ground mobile robots in unstructured environments," Mech. Sci., pp. 49-62, 2012, doi: 10.5194/ms-3-49-2012.

[2] H. Taheri and Z. Chun Xia, "Omnidirectional mobile robots, mechanisms and navigation approaches," Mech. Mach. Theory, vol. 153, no. 103958, 2020, doi: https://doi.org/10.1016/j.mechmachtheory.2020.103958.

[3] B. I. Adamov, "INFLUENCE OF MECANUM WHEELS CONSTRUCTION ON ACCURACY OF THE OMNIDIRECTIONAL PLATFORM NAVIGATION ( ON EXANPLE OF KUKA YOUBOT ROBOT )," 2018 25th Saint Petersbg. Int. Conf. Integr. Navig. Syst., pp. 1-4, 2018.

[4] H. Taheri, B. Qiao, and N. Ghaeminezhad, "Kinematic Model of a Four Mecanum Wheeled Mobile Robot," Int. J. Comput. Appl., vol. 113, no. 3, pp. 6-9, Mar. 2015, doi: $10.5120 / 19804-1586$.

[5] J. Röwekämper, C. Sprunk, G. D. Tipaldi, C. Stachniss, P. Pfaff, and W. Burgard, "On the position accuracy of mobile robot localization based on particle filters combined with scan matching," IEEE/RSJ Int. Conf. Intell. Robot. Syst., 2012, doi: 10.1109/IROS.2012.6385988

[6] R. E. Kalman, "A New Approach to Linear Filtering and Prediction Problems," J. Basic Eng., vol. 82, no. 1, pp. 35-45, 1960, doi: https://doi.org/10.1115/1.3662552.

[7] S. J. Julier and J. K. Uhlmann, "A new extension of the
Kalman filter to nonlinear systems," Int. Soc. Opt. Eng., pp. 182-193, 1997, doi https://doi.org/10.1117/12.280797.

[8] M. Bahraini, M. Bozorg, and A. Rad, "A new adaptive UKF algorithm to improve the accuracy of SLAM," Int J Robot, vol. 5, no. 1, pp. 1-12, 2018.

[9] W. R. Gilks, "Markov Chain Monte Carlo," in Encyclopedia of Biostatistics, 2005.

[10] T. T. Mac, C. Copot, D. T. Tran, and R. De Keyser, "Heuristic approaches in robot path planning: a survey," Rob. Auton. Syst., vol. 86, pp. 13-28, 2016, doi: https://doi.org/10.1016/j.robot.2016.08.001.

[11] M. Oscar, U. Orozco-Rosas, and R. Sep'ulveda, "Path planning for mobile robots using Bacterial Potential Field for avoiding static and dynamic obstacles," Expert Syst. Appl., vol. 42, no. 12, pp. 5177-5191, 2015, doi: https://doi.org/10.1016/j.eswa.2015.02.033.

[12] M. K. Singh and D. R. Parhi, "Path optimisation of a mobile robot using an artificial neural network controller," Int. J. Syst. Sci., vol. 42, no. 1, pp. 107-120, 2011, doi: https://doi.org/10.1080/00207720903470155.

[13] Z. Peng, G. Wen, S. Yang, and A. Rahmani, "Distributed consensus-based formation control for nonholonomic wheeled mobile robots using adaptive neural network," Nonlinear Dyn., vol. 86, no. 1, pp. 605-622, 2016, doi: https://doi.org/10.1007/s11071-016-2910-2.

[14] Q. Zhu, Y. Han, P. Liu, Y. Xiao, P. Lu, and C. Cai, "Motion Planning of Autonomous Mobile Robot Using Recurrent Fuzzy Neural Network Trained by Extended Kalman Filter," Hindawi Comput. Intell. Neurosci., vol. 2019, 2019, doi: https://doi.org/10.1155/2019/1934575.

[15] A. Dorra, B. Maïssa, and R. Chokri, "Hybrid Type-2 Fuzzy-Sliding Mode Controller for Navigation of Mobile Robot in an Environment Containing a Dynamic Target," J. Robot., 2018, doi: https://doi.org/10.1155/2018/8421848.

[16] S. Balaji and S. Karthikeyan, "A survey on moving object tracking using image processing," 2017 11th Int. Conf. Intell. Syst. Control, pp. 469-474, 2017, doi: https://doi.org/10.1109/ISCO.2017.7856037. 